\title{
ON THE SPIRAL-INDUCED NONLINEAR DISSIPATIVE PROCESS TO THE EVOLUTION OF THE GALACTIC DISK
}

\author{
XIAOLEI ZHANG \\ Harvard-Smithsonian Center for Astrophysics \\ 60 Garden St., MS 78, Cambridge, MA 02138, USA
}

\section{Introduction}

It is well known that in dynamical systems governed by long-range forces, the secular evolution of the system is driven predominantly by collective effects. In the current paper, we report the discovery of such a collective dissipation process induced by a self-sustained spiral density wave, which leads to important secular evolution effects in spiral galaxies.

\section{Damping of the Spiral Density Wave Modes}

It is found that in general there exists a phase shift between a self-consistent spiral potential and density pairs, as revealed through both the Poisson equation and the equations of motion. The sense of this phase shift is such that the potential spiral lags the density spiral in the azimuthal direction, for all trailing spirals which have radial density falloff slower than $r^{-3 / 2}$. For a self-sustained spiral potential-density pair, the phase shift changes sign near the corotation radius. Therefore there is a torque exerted by the potential spiral on the density spiral, and a secular transfer of energy and angular momentum from the disk mass to the density wave in the inner disk, causing the wave to be damped. The nonlinear evolution of the wave amplitude follows the Landau equation for nonequilibrium phase transition.

3. Secular Heating of the Galactic Disk and the Origin of AgeVelocity Dispersion Relation in the Solar Neighborhood

Due to the difference in the angular speed of the matter $\Omega$ and the pattern speed of the wave $\Omega_{p}$, the energy released by the disk mass can not be com- 
pletely absorbed by the wave. The rest contributes to the secular heating of the disk. The space velocity diffusion coefficient of such a process is found to be

$$
D=\left(\Omega-\Omega_{p}\right) F^{2} v_{c}^{2} \tan i \sin \left(m \phi_{0}\right)
$$

where $F$ is the fractional amplitude of the spiral wave, $v_{c}$ the circular speed, $i$ the pitch angle of the spiral, $m$ the number of spiral arms, and $\phi_{0}$ the equivalent phase shift. This could quantitatively explain the age-velocity dispersion relation for the solar neighborhood stars.

\section{Secular Radial Mass Accretion and the Building of Disk and Bulge}

The energy and angular momentum transfer from the disk mass to the density wave causes the orbit of disk stars to decay secularly. For a nearly constant orbital decay rate, the partial differential equation which governs the evolution of the disk surface density $\Sigma$ admits the following self-similar solution

$$
\Sigma=\Sigma_{0} \frac{e^{-r / r_{0}}}{r / r_{0}} e^{\frac{-(d r / d t)_{0}}{r_{0}}} t
$$

where $\Sigma_{0}$ and $r_{0}$ are constants, and the orbital decay rate $(d r / d t)_{0}$ is related to the various spiral and disk parameters through

$$
\left(\frac{d r}{d t}\right)_{0}=-\frac{1}{2} F^{2} v_{c} \tan i \sin \left(m \phi_{0}\right)
$$

In (2), the choice $r_{0}=\infty$ describes a time-independent $1 / r$ surface density and a constant radial flux which goes entirely to the center (building the bulge). A finite and large $r_{0}$ corresponds to a slow build up of the disk surface density. On the other hand, (2) with a small value of $r_{0}$ could also describe the self-similar build-up of the bulge itself, since the resulting density is almost identical to the de Vaucouleurs $r^{1 / 4}$ law. For a $20^{\circ}$ pitch angled spiral galaxy with properties similar to our own Galaxy, the orbital decay rate is about $5 \mathrm{kpc}$ in a Hubble time. A bulge size of $10^{10} M_{\odot}$ can thus be built up in a Hubble time.

\section{Evolution Along the Hubble Sequence}

As a result of the radial mass accretion in the inner disk (and excretion outside the corotation), the morphology a the disk galaxy evolves towards that of a high central concentration together with a diffused and extended envelope. The kind of spiral modes admitted by the disk thus also evolve from the open type to that of the more tightly wound. In general, the nonlinear dissipative process discussed in this paper causes a spiral galaxy to evolve along the Hubble sequence from the late to the early types. 